\title{
THE ARGUMENT PRINCIPLE AND HOLOMORPHIC EXTENDIBILITY
}

\author{
Josip Globevnik
}

\begin{abstract}
Let $D$ be a bounded domain in the complex plane whose boundary consists of finitely many pairwise disjoint simple closed curves. Give $b D$ the standard orientation and let $A(D)$ be the algebra of all continuous functions on $\bar{D}$ which are holomorphic on $D$. In the paper we prove that a continuous function $f$ on $b D$ extends to a function in $A(D)$ if and only if for each $g \in A(D)$ such that $f+g \neq 0$ on $b D$ the change of argument of $f+g$ along $b D$ is nonnegative.
\end{abstract}

\section{Introduction and the main result}

H. Alexander and J. Wermer $[\mathrm{AW}]$ obtained a characterization of boundaries of analytic varieties in terms of a generalized argument principle. Their results brought new results also into the classical function theory of one variable. For instance, E. L. Stout [S1] observed that their results imply that a smooth function on the unit circle $b \Delta$ extends holomorphically through the open unit disc $\Delta$ if and only if for each polynomial $Q$ of two variables such that $Q(z, f(z)) \neq 0(z \in b \Delta)$ the change of argument of $z \mapsto Q(z, f(z))$ around $b \Delta$ is nonnegative, and proved that this holds for continuous functions. J. Wermer $[\mathrm{W}]$ showed that for smooth functions on $b \Delta$ there is a better result: a smooth function $f$ on $b \Delta$ extends holomorphically through $\Delta$ if and only if for each polynomial $P$ such that $f+P \neq 0$ on $b \Delta$ the change of argument of $f+P$ around $b \Delta$ is nonnegative. For continuous functions this was proved by the author in [Gl] so that we have the following characterization of the disc algebra in terms of the argument principle:

Theorem 1.0 [Gl] A continuous function $f$ on $b \Delta$ extends holomorphically through $\Delta$ if and only if for each polynomial $P$ such that $f+P \neq 0$ on $b \Delta$ the change of argument of $f+P$ around $b \Delta$ is nonnegative.

In the present paper we prove that the analogous theorem holds for multiply connected domains.

Let $D \subset \mathbb{C}$ be a bounded domain whose boundary consists of finitely many pairwise disjoint simple closed curves. We give $b D$ the standard orientation. Denote by $A(D)$ the algebra of all continuous functions on $\bar{D}$ which are holomorphic on $D$. Our main result is

THEOREM 1.1 A continuous function $f$ on $b D$ extends to a function in $A(D)$ if and only if for each $g \in A(D)$ such that $f+g \neq 0$ on $b D$ the change of argument of $f+g$ along $b D$ is nonnegative.

If the condition in Theorem 1.1 holds for all $g$ belonging to a dense subset of $A(D)$ then it holds for all $g \in A(D)$. Thus, since rational functions with poles outside $\bar{D}$ are dense in $A(D)$ [S2, 23], it is enough to assume that the condition in Theorem 1.1 holds for rational functions with poles outside $\bar{D}$. The only if part of the theorem is an obvious consequence of the argument principle. In fact, if $f$ admits an extension $\tilde{f} \in A(D)$ then 
the change of argument of $f+g$ along $b D$ equals $2 \pi$ times the number of zeros of $\tilde{f}+g$ in $D$.

\section{Preliminaries}

Every bounded domain $D \subset \mathbb{C}$ whose boundary consists of finitely many pairwise disjoint simple closed curves is biholomorphically equivalent to a domain $D^{\prime}$ bounded by finitely many pairwise disjoint circles [Go]. Moreover, every biholomorphic map $\Phi: D \rightarrow D^{\prime}$ extends to a homeomorphism $\tilde{\Phi}: \bar{D} \rightarrow \overline{D^{\prime}}$ (see the proof in [CL, pp. 46-49] which works also for multiply connected domains, bounded by finitely many pairwise disjoint simple closed curves). Thus, with no loss of generality assume that $D$ is bounded by finitely many pairwise disjoint circles.

In general, not every real-valued harmonic function $u$ on $D$ is the real part of a holomorphic function on $D$. If there is a harmonic function $v$ on $D$ such that $u+i v$ is holomorphic on $D$ then we call $v$ a conjugate of $u$. If $D$ is simply connected then every harmonic function on $D$ has a conjugate on $D$. Let $f$ be a complex valued harmonic function on $D$. Write $f=p+i q$ with $p, q$ real. We will say that $f$ has a conjugate on $D$ if $p$ has a conjugate $r$ on $D$ and $q$ has a conjugate $s$ on $D$, and we will call the function $r-i s$ a conjugate of $f$ on $D$. This happens if and only if $f=F+\bar{G}$ where $\mathrm{F}$ and $\mathrm{G}$ are holomorphic functions on $D$. In fact, if $P=p+i r$ and $Q=q+i s$ then $P$ and $Q$ are holomorphic functions on $D$ and $F=(P+i Q) / 2$ and $G=(P-i Q) / 2$.

Given a continuous function $\Phi$ on $b D$ there is a continuous extension of $\Phi$ to $\bar{D}$ which is harmonic on $D$ and which we will denote by $\mathcal{H}(\Phi)$; moreover, if $\Phi \in C^{\infty}(b D)$ then $\mathcal{H}(\Phi) \in C^{\infty}(\bar{D})[\mathrm{B}, \mathrm{p} .53]$. $\mathcal{H}$ is a linear map from $C(b D)$ to the space of continuous functions on $\bar{D}$ which are harmonic on $D$. If $\mathrm{D}$ is simply connected then $\mathcal{H}(\Phi)$ has a conjugate harmonic function on $D$ which is also in $C^{\infty}(\bar{D})$ provided that $\Phi \in C^{\infty}(b D)[\mathrm{B}$, p.91]. If $\Omega_{1}$ and $\Omega_{2}$ are bounded, simply connected domains with boundaries of class $C^{\infty}$ then a biholomorphic map $\Phi$ from $\Omega_{1}$ to $\Omega_{2}$ extends to a smooth map from $\overline{\Omega_{1}}$ to $\overline{\Omega_{2}}$ [B, p.28]. Applying this locally along $b D$ and using the preceding discussion we see that if $f$ is a harmonic function on $D$ that has a conjugate on $\mathrm{D}$ then the conjugate extends smoothly to $\bar{D}$ provided that $f$ extends smoothly to $\bar{D}$. We summarize this in

LEMMA 2.1 If $f \in C^{\infty}(b D)$ is such that $\mathcal{H}(f)$ has a conjugate on $D$ then both $\mathcal{H}(f)$ and its conjugate extend smoothly to $\bar{D}$.

Every harmonic function $f$ on $D$ is real-analytic on $D$ so, if $f$ is holomorphic on an open subset of $D$ then it is holomorphic on $D$. We will need the following fact which can be found as an exercise in $[\mathrm{R}]$.

LEMMA 2.2 Let $f$ be a harmonic function on $D$ such that $z \mapsto z f(z)$ is harmonic on a nonempty open set $U \subset D$. Then $f$ is holomorphic on $D$.

Proof. By the preceding discussion it is enough to prove that $f$ is holomorphic on a $\operatorname{disc} \Omega \subset D$. Since $f$ is harmonic on $D$ there is a disc $\Omega \subset U$ such that $f=P+\bar{Q}$ on $\Omega$ where $P$ and $Q$ are holomorphic functions on $\Omega$. By our assumption, the function 
$z \mapsto z f(z)=z P(z)+z \overline{Q(z)}$ is harmonic on $\Omega$ so

$$
\frac{\partial^{2}}{\partial z \partial \bar{z}}[z P(z)+z \overline{Q(z)}]=0 \quad(z \in \Omega)
$$

which implies that $\overline{Q^{\prime}(z)}=0(z \in \Omega)$. Thus, $Q$ is constant on $\Omega$ and consequently $f$ is holomorphic on $\Omega$. This completes the proof.

\section{A new proof in the case of a disc}

The proof in [Gl] does not generalize to multiply connected domains. In this section we give a new, different proof of the theorem in the case when $D$ is a disc which we later generalize to multiply connected domains.

Write $\Delta=\{\zeta \in \mathbb{C}:|\zeta|<1\}$. Throughout this section, $D=\Delta$, Denote by $Z$ the identity function: $Z(z)=z(z \in b D)$. Assume that $f \in C(b \Delta)$ does not extend to a function from the disc algebra $A(\Delta)$. Then there is an $a \in \Delta$ such that

$$
\mathcal{H}[(Z-a) f](a) \neq 0 .
$$

To see this, suppose for a moment that $\mathcal{H}[(Z-a) f](a)=0(a \in \Delta)$. This implies that $\mathcal{H}(Z f)(a)=a \mathcal{H}(f)(a)(a \in \Delta)$. In particular, the function $a \mapsto a \mathcal{H}(f)(a)$ is harmonic on $\Delta$. Since $\mathcal{H}(f)$ is harmonic on $D$ Lemma 2.2 implies that $\mathcal{H}(f)$ is holomorphic on $\Delta$ so $f$ extends holomorphically through $\Delta$, a contradiction. This proves that there is an $a \in \Delta$ such that (3.1) holds.

With no loss of generality, replacing $f$ with $e^{i \omega} f, \omega \in \mathbb{R}$, if necessary, we may assume that

$$
\Re\{\mathcal{H}[(Z-a) f](a)\}=\beta \neq 0 .
$$

For easier understanding we complete the proof first under the additional assumption that $f$ is smooth. Suppose for a moment that $f$ is smooth. The function $z \mapsto \Re\{\mathcal{H}[(Z-$ a) $f](z)\}-\beta$ is continuous on $\bar{\Delta}$, harmonic on $\Delta$ and has smooth boundary values $\Re[(z-$ a) $f(z)]-\beta(z \in b \Delta)$. Hence by Lemma 2.1 there is a function $g \in A(\Delta)$ such that

$$
\Re[g(z)]=\Re\{\mathcal{H}[(Z-a) f](z)\}-\beta \quad(z \in \bar{\Delta}) .
$$

By (3.2) we have $\Re g(a)=\Re\{\mathcal{H}[(Z-a) f](a)\}-\beta=0$ so by adding an imaginary constant to $g$ if necessary we may assume that $g(0)=0$ so $g(z)=(z-a) h(z)(z \in \bar{\Delta})$ where $h \in A(\Delta)$. Consider the function $z \mapsto G(z)=(z-a) f(z)-g(z)(z \in b \Delta)$. We have $\Re[G(z)]=\beta(z \in b \Delta)$ which, since $\beta \neq 0$, implies that $G \neq 0$ on $b \Delta$ and that the change of argument of $G$ around $b \Delta$ equals zero. Since $a \in \Delta$ the change of argument of $z \mapsto(z-a)$ around $b \Delta$ equals $2 \pi$. Since $G(z)=(z-a)[f(z)-h(z)](z \in b \Delta)$ it follows that $f-h \neq 0$ on $b \Delta$ and that the change of argument of $f-h$ around $b \Delta$ is negative. Since $h \in A(\Delta)$ this completes the proof in the special case when $f$ is smooth.

In general, we have to approximate $f$ by smooth functions as follows:

Let $f_{1}$ be a smooth function on $b \Delta$ such that

$$
\left|(z-a)\left[f(z)-f_{1}(z)\right]\right|<|\beta| / 4 \quad(z \in b \Delta) .
$$


Write $\beta_{1}=\Re\left\{\mathcal{H}\left[(Z-a) f_{1}\right](a)\right\}$. By (3.3) and by the maximum principle for the real harmonic function $\Re\left\{\mathcal{H}\left[(Z-a)\left(f_{1}-f\right)\right]\right\}$ we have

$$
\left|\beta_{1}-\beta\right|<|\beta| / 4 \text {. }
$$

The function $z \mapsto \Re\left\{\mathcal{H}\left[(Z-a) f_{1}\right](z)\right\}-\beta_{1}$ is continuous on $\bar{D}$, harmonic on $\Delta$ and has smooth boundary values $\Re\left[(z-a) f_{1}(z)\right]-\beta_{1}(z \in b \Delta)$. Hence by Lemma 2.1 there is a function $g_{1} \in A(\Delta)$ such that

$$
\Re\left[g_{1}(z)\right]=\Re\left\{\mathcal{H}\left[(Z-a) f_{1}\right](z)\right\}-\beta_{1}(z \in \bar{\Delta}) .
$$

Clearly $\Re\left[g_{1}(a)\right]=\Re\left\{\mathcal{H}\left[(Z-a) f_{1}\right](a)\right\}-\beta_{1}=0$ so by adding an imaginary constant to $g_{1}$ if necessary we may assume that $g_{1}(a)=0$ so that $g_{1}(z)=(z-a) h(z)(z \in \bar{\Delta})$ where $h \in A(\Delta)$.

Consider the function $z \mapsto G(z)=(z-a) f(z)-g_{1}(z)(z \in b \Delta)$. By (3.5) we have $\Re G(z)=\beta+\Re\left[(z-a)\left(f(z)-f_{1}(z)\right)\right]+\left(\beta_{1}-\beta\right)(z \in b \Delta)$ which, by (3.3) and (3.4) implies that

$$
|\Re G(z)-\beta|<|\beta| / 4+|\beta| / 4=|\beta| / 2 \quad(z \in b \Delta)
$$

so

$$
\beta-|\beta| / 2<\Re G(z)<\beta+|\beta| / 2(z \in \Delta),
$$

which, since $\beta \neq 0$, implies that $G \neq 0$ on $b \Delta$ and that the change of argument of $G$ around $b \Delta$ is zero. We now repeat the reasoning from the proof in the smooth case to conclude that $f-h \neq 0$ on $b \Delta$ and that the change of argument of $f-h$ around $b \Delta$ is negative. Since $h \in A(\Delta)$ this completes the proof.

\section{Harmonic functions and their conjugates}

The main problem in generalizing the proof in Section 3 to multiply connected domains $D$ is that in general, a harmonic function on $D$ has no conjugate on $D$.

We have assumed that $D \subset \subset \mathbb{C}$ is a domain bounded by pairwise disjoint circles. Denote these circles by $\Gamma_{1}, \Gamma_{2}, \cdots, \Gamma_{n}$ where $\Gamma_{n}$ is the boundary of the unbounded component of $\mathbb{C} \backslash \bar{D}$. For each $k, 1 \leq k \leq n$, the harmonic measure function $\omega_{k}$ is the continuous function on $\bar{D}$, harmonic on $D$ which satisfies $\omega_{k} \equiv 1$ on $\Gamma_{k}$ and $\omega_{k} \equiv 0$ on $\Gamma_{j}, 1 \leq j \leq n, j \neq k$. By the preceding discussion each $\omega_{k}, 1 \leq k \leq n$, is smooth on $\bar{D}$. We have $\sum_{k=1}^{n} \omega_{k} \equiv 1$ on $\bar{D}$.

For each $k, 1 \leq k \leq n-1$, let $\gamma_{k}$ be a circle with the same center as $\Gamma_{k}$ and with a a slightly larger radius, and let $\gamma_{n}$ be a circle with the same center as $\Gamma_{n}$ and with a slightly smaller radius so that the circles $\gamma_{k}, 1 \leq k \leq n$ bound a domain $D^{\prime}$, slightly smaller than $D$, whose closure is contained in $D$. We give each $\gamma_{k}$ the orientation induced by the standard orientation of $b D^{\prime}$.

Let $u$ be a real-valued harmonic function on $D$. A conjugate $v$ of $u$ has to satisfy the Cauchy-Riemann equations

$$
\frac{\partial v}{\partial x}=-\frac{\partial u}{\partial y} \quad \frac{\partial v}{\partial y}=\frac{\partial u}{\partial x}
$$


This system is always solvable for $v$ locally. It is solvable for $v$ on $D$ if and only if

$$
\int_{\gamma_{k}}\left(-\frac{\partial u}{\partial y} d x+\frac{\partial u}{\partial x} d y\right)=0 \quad(1 \leq k \leq n-1) .
$$

Since

$$
2 \int_{\gamma_{k}} \frac{\partial u}{\partial z} d z=i \int_{\gamma_{k}}\left(-\frac{\partial u}{\partial y} d x+\frac{\partial u}{\partial x} d y\right) \quad(1 \leq k \leq n-1)
$$

(4.1) holds if and only if

$$
\int_{\gamma_{k}} \frac{\partial u}{\partial z} d z=0 \quad(1 \leq k \leq n-1)
$$

If $u$ is a real valued harmonic function on $D$ then there are real constants $c_{1}, c_{2}, \cdots, c_{n-1}$ such that the harmonic function $u+\sum_{j=1}^{n-1} c_{j} \omega_{j}$ has a conjugate on $D$. For this to happen we must have

$$
\int_{\gamma_{k}} \frac{\partial}{\partial z}\left[u+\sum_{j=1}^{n-1} c_{j} \omega_{j}\right] d z=0 \quad(1 \leq k \leq n-1)
$$

that is,

$$
\sum_{j=1}^{n-1} c_{j} \int_{\gamma_{k}} \frac{\partial \omega_{j}}{\partial z} d z=-\int_{\gamma_{k}} \frac{\partial u}{\partial z} d z
$$

The system (4.3) has a unique solution since the matrix

$$
\left[\int_{\gamma_{k}} \frac{\partial \omega_{j}}{\partial z} d z\right]_{1 \leq j, k \leq n-1}=\left[\int_{\Gamma_{k}} \frac{\partial \omega_{j}}{\partial z} d z\right]_{1 \leq j, k \leq n-1}
$$

is known to be nonsingular [B, p.82]. (In the last equality we used the fact that all functions $\frac{\partial \omega_{j}}{\partial z}$ are smooth on $\bar{D}$ and holomorphic on $D$.) The Poisson formula implies that given $\varepsilon>0$ and a compact set $K \subset D$ there is a $\delta>0$ such that $\left|\frac{\partial u}{\partial z}\right|<\varepsilon$ on $K$ whenever $u$ is a real harmonic function on $D$ such that $|u|<\delta$ on $D$. Thus, given $\varepsilon>0$ there is a $\delta>0$ such that

$$
\left|\int_{\gamma_{k}} \frac{\partial u_{1}}{\partial z} d z-\int_{\gamma_{k}} \frac{\partial u}{\partial z} d z\right|<\varepsilon \quad(1 \leq k \leq n-1)
$$

provided that $u_{1}$ is a real harmonic function on $D$ satisfying $\left|u_{1}(z)-u(z)\right|<\delta(z \in D)$.

The preceding discussion gives

LEMMA 4.1 Given a harmonic function $f$ on $D$ there is a unique $(n-1)$-tuple $c_{1}(f), \cdots$, $c_{n-1}(f)$ of complex numbers such that $f+\sum_{j=1}^{n-1} c_{j}(f) \omega_{j}$ has a conjugate on $D$. These numbers depend continuously on $f$ in the sup norm on $D$.

\section{Proof of Theorem 1.1, Part 1}

Let $D$ be a domain bounded by pairwise disjoint circles $\Gamma_{1}, \cdots, \Gamma_{n}$ where $\Gamma_{n}$ is the boundary of the unbounded component of $\mathbb{C} \backslash \bar{D}$. Section 3 contains the proof of Theorem 1.1 in the case $n=1$ so assume that $n \geq 2$. 
Let $f$ be a continuous function on $b D$ which does not extend holomorphically through D. Define

$$
A(a, f)=\mathcal{H}[(Z-a) f](a)=\mathcal{H}(Z f)(a)-a \mathcal{H}(f)(a) \quad(a \in \bar{D}) .
$$

Since $\mathcal{H}(f)$ is not holomorphic Lemma 2.2 implies that

$$
\{a \in D: A(a, f)=0\} \text { is a closed, nowhere dense subset of } D .
$$

There are constants $c_{k}(f), 1 \leq k \leq n-1$, such that

$$
\mathcal{H}(f)(z)+\sum_{k=1}^{n-1} c_{k}(f) \omega_{k}(z)=P_{f}(z)+\overline{Q_{f}(z)} \quad(z \in D)
$$

where $P_{f}$ and $Q_{f}$ are holomorphic functions on $D$. Similarly, there are constants $d_{k}(f), 1 \leq$ $k \leq n-1$, such that

$$
\mathcal{H}(Z f)(z)+\sum_{k=1}^{n-1} d_{k}(f) \omega_{k}(z)=R_{f}(z)+\overline{S_{f}(z)} \quad(z \in D)
$$

where $R_{f}$ and $S_{f}$ are holomorphic functions on $D$. We know that the constants $c_{k}(f)$ and $d_{k}(f), 1 \leq k \leq n-1$, are determined uniquely and, by the maximum principle for harmonic functions depend continuously on $f \in C(b D)$. We have

$$
c_{k}\left(e^{i \omega} f\right)=e^{i \omega} c_{k}(f), \quad d_{k}\left(e^{i \omega} f\right)=e^{i \omega} d_{k}(f) \quad(1 \leq k \leq n-1, \omega \in \mathbb{R})
$$

and

$$
A\left(a, e^{i \omega} f\right)=e^{i \omega} A(a, f) \quad(\omega \in \mathbb{R}, a \in D) .
$$

Define

$$
\Phi_{a, f}(z)=\sum_{j=1}^{n-1}\left[d_{j}(f)-a c_{j}(f)\right] \cdot\left[\omega_{j}(z)-\omega_{j}(a)\right]-A(a, f) \quad(z \in D) .
$$

For each $a \in D$ the function $\Phi_{a, f}$ is smooth on $\bar{D}$ and harmonic on $D$. By the preceding discussion for each $a \in D$ the harmonic function

$$
z \mapsto \mathcal{H}[(Z-a) f](z)+\Phi_{a, f}(z) \quad(z \in D)
$$

vanishes at $a$ and has a conjugate on $D$, that is, it is of the form $F_{a, f}+\overline{G_{a, f}}$ where $F_{a, f}$ and $G_{a, f}$ are holomorphic on $D$.

\section{The function $\Phi_{a, f}$}

Note that for each $a \in D$ the function $\Phi_{a, f}$ is smooth on $\bar{D}$, harmonic on $D$ and constant on each component $\Gamma_{j}, 1 \leq j \leq n$, of $b D$.

LEMMA 6.1 There is an $a \in D$ such that $\Phi_{a, f}(z) \neq 0(z \in b D)$. 
Proof. Recall that for each $a \in D$ the function $\Phi_{a, f} \mid \Gamma_{k}$ is constant for each $k, 1 \leq k \leq n$; we have to prove that for some $a \in D$ these constants are all different from 0 . We shall prove that

$$
\left.\begin{array}{l}
\text { for each } k, 1 \leq k \leq n, \text { the set }\left\{a \in D: \Phi_{a, f} \mid \Gamma_{k}=0\right\} \\
\text { is a closed subset of } D \text { with empty interior. }
\end{array}\right\}
$$

Assume that we have done this. Then $\cup_{k=1}^{n}\left\{a \in D: \Phi_{a, f} \mid \Gamma_{k}=0\right\}$ is a closed subset of $D$ with empty interior which implies that there is an open dense subset of $D$ of those $a$ for which $\Phi_{a, f}(z) \neq 0(z \in b D)$ which will complete the proof. It remains to prove (6.1).

Let $1 \leq k \leq n-1$. On $\Gamma_{k}$ the function $\Phi_{a, f}$ is equal to the constant $-A(a, f)+$ $\sum_{j=1, j \neq k}^{n-1}\left[d_{j}(f)-a c_{j}(f)\right] \cdot\left[-\omega_{j}(a)\right]+\left[d_{k}(f)-a c_{k}(f)\right] .\left[1-\omega_{k}(a)\right]$. Since $a \mapsto A(a, f)$ is continuous on $D$ it follows that $\left\{a \in D: \Phi_{a, f} \mid \Gamma_{k}=0\right\}$ is a closed subset of $D$. Suppose that it contains a $\operatorname{disc} U$. Then

$$
A(a, f)=\sum_{j=1, j \neq k}^{n-1}\left[d_{j}(f)-a c_{j}(f)\right] \cdot\left[-\omega_{j}(a)\right]+\left[d_{k}(f)-a c_{k}(f)\right] \cdot\left[1-\omega_{k}(a)\right]
$$

for all $a \in U$. Since both sides of (6.2) are real-analytic in $a$ on $D$ it follows that (6.2) holds for all $a \in D$. Since both sides of (6.2) are continuous in $a$ on $\bar{D}$ it follows that (6.2) holds for all $a \in b D$. However, $A(a, f)=0(a \in b D)$ so

$$
\sum_{j=1, j \neq k}^{n-1}\left[d_{j}(f)-a c_{j}(f)\right] \cdot\left[\omega_{j}(a)\right]=\left[d_{k}(f)-a c_{k}(f)\right] .\left[1-\omega_{k}(a)\right] \quad(a \in b D) .
$$

If $a \in \Gamma_{j}, 1 \leq j \leq n-1, j \neq k$, then $\omega_{j}(a)=1$ and $\omega_{i}(a)=0$ for all $i, 1 \leq i \leq n, i \neq j$, so (6.3) implies that

$$
d_{j}(f)-a c_{j}(f)=d_{k}(f)-a c_{k}(f) \quad\left(a \in \Gamma_{j}, 1 \leq j \leq n-1, j \neq k\right) .
$$

If $a \in \Gamma_{n}$ then $\omega_{j}(a)=0$ for all $j, 1 \leq j \leq n-1$, including $k$, so (6.3) gives

$$
d_{k}(f)-a c_{k}(f)=0 \quad\left(a \in \Gamma_{n}\right) .
$$

Now, (6.5) implies that $d_{k}(f)=c_{k}(f)=0$ which, by (6.4) gives $d_{j}(f)=c_{j}(f)=0(1 \leq$ $j \leq n-1, j \neq k)$ so, by $(6.2)$ it follows that $A(a, f)=0$ for every $a \in D$ which contradicts (5.1). This proves that $\left\{a \in D: \Phi_{a, f} \mid \Gamma_{k}=0\right\}$ has empty interior for each $k, 1 \leq k \leq n-1$.

Let $k=n$. We have

$$
\Phi_{a, f} \mid \Gamma_{n}=-A(a, f)+\sum_{j=1}^{n-1}\left[d_{j}(f)-a c_{j}(f)\right] \cdot\left[-\omega_{j}(a)\right]
$$

As before, the continuity of $a \mapsto A(a, f)$ on $D$ implies that the set $\left\{a \in D: \Phi_{a, f} \mid \Gamma_{n}=0\right\}$ is closed. Suppose that it has nonempty interior. As before, we get

$$
0=A(a, f)=\sum_{j=1}^{n-1}\left[d_{j}(f)-a c_{j}(f)\right] \cdot\left[-\omega_{j}(a)\right] \quad(a \in b D)
$$


It follows that $d_{j}(f)-a c_{j}(f)=0 \quad\left(a \in \Gamma_{j}, 1 \leq j \leq n-1\right\}$ which implies that $d_{j}(f)=$ $c_{j}(f)=0 \quad(1 \leq j \leq n-1)$ so again $A(a, f) \equiv 0(a \in D)$ which contradicts (5.1). This completes the proof.

\section{Proof Theorem 1.1, Part 2}

By Lemma 6.1 there is an $a \in D$ such that the constants $\Phi_{a, f} \mid \Gamma_{k}, 1 \leq k \leq n$, are all different from 0. By (5.4) and (5.5) we have $\Phi_{a, e^{i \omega} f}=e^{i \omega} \Phi_{a, f}(\omega \in \mathbb{R})$ so replacing $f$ by $e^{i \omega} f$ if necessary we may assume with no loss of generality that

$$
\left(\Re \Phi_{a, f}\right) \mid \Gamma_{k}=\beta_{k} \neq 0 \quad(1 \leq k \leq n) .
$$

To make the proof easier to understand we first complete it under the assumption that $f$ is smooth. Assume that $f$ is smooth. In this case, by Lemma 2.1,

$$
\mathcal{H}[(Z-a) f](z)+\Phi_{a, f}(z)=F_{a, f}+\overline{G_{a, f}(z)} \quad(z \in \bar{D})
$$

where $F_{a, f}$ and $G_{a, f}$ belong to $A(D)$ so

$$
\Re\left\{\mathcal{H}[(Z-a) f](z)+\Phi_{a, f}(z)\right\}=\Re[g(z)] \quad(z \in \bar{D})
$$

where $g=\left(F_{a, f}+G_{a, f}\right) / 2 \in A(D)$. Clearly $\Re[g(a)]=0$ so by adding an imaginary constant to $g$ if necessary we may assume that $g(0)=0$ so $g(z)=(z-a) h(z)(z \in \bar{D})$ where $h \in A(D)$.

Consider the function $z \mapsto G(z)=(z-a) f(z)-g(z)(z \in b D)$. We have $\Re[G(z)]=$ $-\Re \Phi_{a, f}(z) \quad(z \in b D)$. By (7.1) for each $k, 1 \leq k \leq n$, the expression on the right is a nonzero constant on $\Gamma_{k}$ which implies that for each $k, 1 \leq k \leq n$, the change of argument of $z \mapsto G(z)=(z-a)[f(z)-h(z)]$ along $\Gamma_{k}$ equals 0 . Since $a \in D$ the change of argument of $z \mapsto(z-a)$ along each $\Gamma_{k}, 1 \leq k \leq n-1$, is zero, and the change of argument of $z \mapsto(z-a)$ along $\Gamma_{n}$ is $2 \pi$. Thus, the change of argument of $z \mapsto f(z)-h(z)$ along $\Gamma_{n}$ equals $-2 \pi$. So, the change of argument of $z \mapsto f(z)-h(z)$ along $b D$ is negative. Since $h \in A(D)$ this completes the proof in the case when $f$ is smooth.

In the case of general $f$ we have to approximate $f$ by smooth functions. We already know that the constants $c_{k}(f)$ and $d_{k}(f)$ depend continuously on $f \in C(b D)$. Further, for our fixed $a \in D$ the maximum priciple for harmonic functions implies that $A(a, f)=$ $\mathcal{H}[(Z-a) f](a)$ also depends continuously on $f \in C(b D)$. It follows that $\Phi_{a, f_{1}}$ is uniformly arbitrarily close to $\Phi_{a, f}$ on $\bar{D}$ provided that $f_{1} \in C(b B)$ is sufficiently close to $f$. Fix $\varepsilon$,

$$
0<\varepsilon<(1 / 4) \min \left\{\left|\beta_{k}\right|: 1 \leq k \leq n\right\}
$$

and let $f_{1}$ be a smooth function on $b D$ which is so close to $f$ that

$$
\left|\Phi_{a, f_{1}}(z)-\Phi_{a, f}(z)\right|<\varepsilon \quad(z \in \bar{D})
$$

and

$$
\left|(z-a)\left[f_{1}(z)-f(z)\right]\right|<\varepsilon \quad(z \in b D) .
$$


As before, since $f_{1}$ is smooth, Lemma 2.1 applies to show that

$$
\Re\left\{\mathcal{H}\left[(Z-a) f_{1}\right](z)+\Phi_{a, f_{1}}(z)\right\}=\Re[g(z)] \quad(z \in \bar{D})
$$

where $g \in A(D)$ satisfies $g(a)=0$. Consider the function $z \mapsto G(z)=(z-a) f(z)-g(z)$. We have $\Re[G(z)]=\Re\left\{(z-a)\left[f(z)-f_{1}(z)\right]\right\}+\Re\left[\Phi_{a, f}(z)\right]+\Re\left[\Phi_{a, f_{1}}(z)-\Phi_{a, f}(z)\right]$ so by (7.4) and (7.5) it follows that

$$
\left|\Re[G(z)]-\Re\left[\Phi_{a, f}(z)\right]\right|<2 \varepsilon \quad(z \in b D)
$$

so by (7.1) it follows that

$$
\left|\Re[G(z)]-\beta_{k}\right|<2 \varepsilon \quad\left(z \in \Gamma_{k}, 1 \leq k \leq n\right),
$$

which, by (7.3), implies that

$$
\beta_{k}-\left|\beta_{k}\right| / 2<\Re[G(z)]<\beta_{k}+\left|\beta_{k}\right| / 2 \quad\left(z \in \Gamma_{k}, 1 \leq k \leq n\right) .
$$

Since $\beta_{k} \neq 0(1 \leq k \leq n)$, it follows that for each $k, 1 \leq k \leq n$, the change of argument of $G$ along $\Gamma_{k}$ is zero. Now we conclude the proof as in the smooth case.

Acknowledgement A part of the work whose results are presented here was done during the author's visit at the University of Oslo in November 2003. The author is grateful to Erik Løw and Niels Øvrelid for their hospitality.

This work was supported in part by the Ministry of Education, Science and Sport of Slovenia through research program Analysis and Geometry, Contract No. P1-0291. 


\section{REFERENCES}

[AW] H. Alexander and J. Wermer: Linking numbers and boundaries of varieties. Ann. Math. 151 (2000) 125-150

[B] S. Bell: The Cauchy transform, Potential Theory, and Conformal Mapping. CRC Press, Boca Raton, 1992

[CL] E. F. Collingwood and A.J.Lohwater: The Theory of Cluster Sets. Cambridge University Press, Cambridge 1966

[Gl] J. Globevnik: Holomorphic extendibility and the argument principle.

To appear in "Complex Analysis and Dynamical Systems II (Proceedings of a conference held in honor of Professor Lawrence Zalcman's sixtieth birthday in Nahariya, Israel, June 9-12, 2003)", Contemp. Math. http://arxiv.org/abs/math.CV/0403446

[Go] G. M. Goluzin: Geometrische Funktionentheorie. VEB Deutscher Verlag der Wissenschaften, Berlin 1957

[R] W. Rudin: Real and Complex Analysis. McGraw-Hill, New York, 1970

[S1] E. L. Stout: Boundary values and mapping degree.

Michig. Math. J. 47 (2000) 353-368

[S2] E. L. Stout: The Theory of Uniform Algebras. Bogden and Quigley, Tarrytown -onHudson, N.Y. 1971

[W] J. Wermer: The argument principle and boundaries of analytic varieties.

Oper. Theory Adv. Appl., 127, Birkhauser, Basel, 2001, 639-659

Institute of Mathematics, Physics and Mechanics

University of Ljubljana

Ljubljana, Slovenia

josip.globevnik@fmf.uni-lj.si 